\title{
Guessing tangents in normal flows
}

Markussen, Bo; Sporring, Jon; Erleben, Kenny

Published in:

Journal of Mathematical Imaging and Vision

DOI:

10.1007/s10851-008-0082-5

Publication date:

2008

Document version

Early version, also known as pre-print

Citation for published version (APA):

Markussen, B., Sporring, J., \& Erleben, K. (2008). Guessing tangents in normal flows. Journal of Mathematical Imaging and Vision, 31(2-3), 195-205. https://doi.org/10.1007/s10851-008-0082-5 


\title{
Guessing Tangents in Normal Flows
}

\author{
Bo Markussen · Jon Sporring • Kenny Erleben
}

Published online: 1 May 2008

(C) Springer Science+Business Media, LLC 2008

\begin{abstract}
Given a flow field parallel to isophote normals, a normal flow field, we seek a unobservable tangential field as the minimum of a general energy functional of the total field. We generalize existing methods to any linear, differential operator order on the combined field while keeping the projection onto the isophote normal constant. We discuss invariant flow fields, present a novel iterative solution based on Euler-Lagrange equations, prove continuous convergence, and give synthetic examples for common energy functionals. Possible uses are: estimating physical flow in image sequences, estimating human growth processes, and co-warping textures in animation sequences.
\end{abstract}

Keywords Optical flow - Generalized energy minimization - Euler-Lagrange $\cdot$ Aperture problem .

Texture morphing

\section{Introduction}

Shape deformation and optical flow often relies on a normal flow. However, normal flow often has a loose relation to 'physical' flow, see Fig. 1. The figure shows two snapshots of a deformation of a circle into an ellipse, and the lines indicate the normals, at which a shape flow may occur. It is evident, that the local metric on the circle is not uniformly scaled, and for computer graphics application, this

\section{B. Markussen}

Dept. of Natural Sciences, University of Copenhagen,

Thorvaldsensvej 40, 1871 Frederiksberg C, Denmark

J. Sporring $(\bowtie) \cdot$ K. Erleben

Dept. of Computer Science, University of Copenhagen,

Universitetsparken 1, 2100 Copenhagen, Denmark

e-mail: sporring@diku.dk implies that a texture deformed along the flow will be unevenly stretched as illustrated by the unevenly deformed texture of the Lena image. Similarly, in optical flow, the flow may only be measured normal to isophotes. For a square moving along a diagonal, such as depicted in Fig. 2(left), the observable, normal flow seems to indicate, that the box is deforming, and is even undefined at sharp corners. A typical solution both in morphing and optical flow is to select a smooth flow such as indicated at the bottom right corner of Fig. 2(right).

Normal flow is a projection of the physical flow onto the isophote normal, and in the present article, we propose to guess the tangential component by a general energy minimization formulation for any linear differential operator. The result of our work inside a squarish, soft window is demonstrated in Fig. 3. The figure shows two different vector fields, left being a vector field with one critical point as generated by the gradients near a maximum, and right being the superposition of normal flow fields of a box moving diagonally, which generates a critical edge. The first case is left unchanged by our method, and the later case results in a diagonally dominated vector field, which fits well with the presupposed box moving diagonally.

\subsection{Related Works}

The majority of work related to deformations via flow fields fall in three categories: warping, morphing, and optical flow.

Warping has recently received a lot of attention for registration and modeling flow. In [1] is given a method for finding the simplest deformation field between two three dimensional shapes by a method of Gaussian smoothing of the components of the vector field, that deforms one object to another, followed by a projection back onto the second object. In [2], the large deformation diffeomorphisms are 

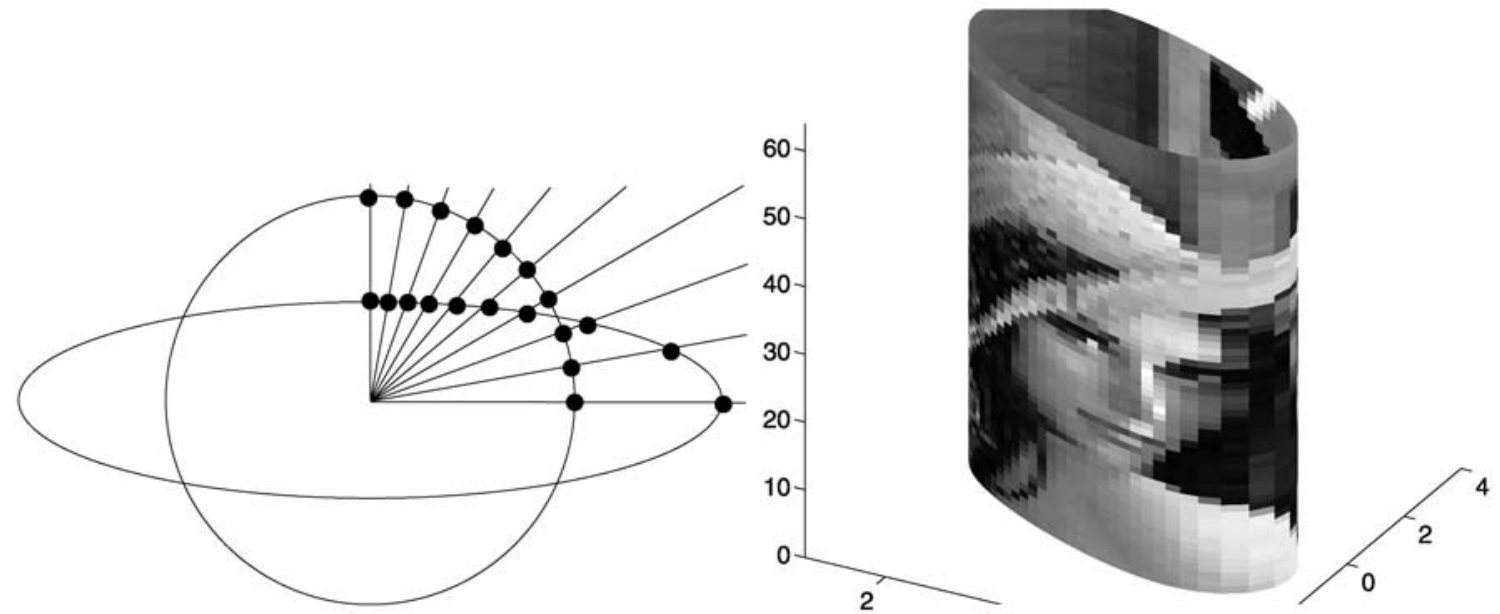

Fig. 1 Warping along normals stretches the curves in an uneven manner
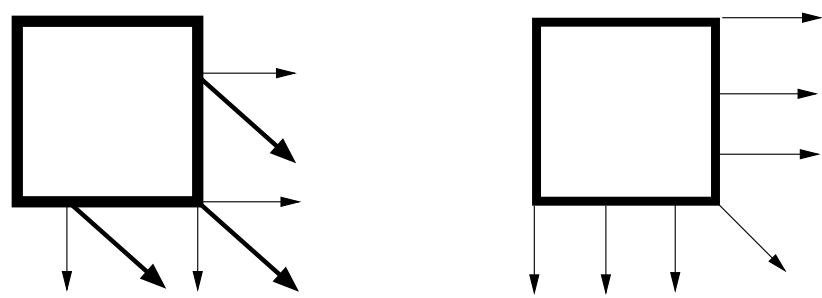

Fig. 2 Left: A black box is moved diagonally implying a diagonal, physical flow on every point (thick arrows). Due to the aperture problem, only the normal flow is observable (thin arrows). Right: A typical solution in Morphing and Optical flow-smooth solution

generated for landmark matching as solutions to the transport equation, which has been applied to e.g. landmark registration in medical applications [3]. The diffeomorphisms in [2] have later been interpreted as the solution to a statistical problem, [4] with application among other things to optical flow [5].

Morphing is an application in computer graphics for changing one shape into another. Many applications are based on level-set methods on signed-distance maps [6, 7]. Most noteworthy is [8], which is based on driving the source signed distance map towards the target signed distance map. This is done by walking in the normal direction of the interface with a distance proportional to the target signed distance map value. In [9] a level set approach for morphing between signed distance maps is presented. Here signed distance maps are constructed from a characteristic scan conversion algorithm, and a similarity measure is formulated as a functional. Differentiating the functional wrt. variation results in a PDE, the solution of the PDE yields the optimal similarity measure. In [10] an efficient numerical narrowband method is presented.

Optical flow is the process of inferring the apparent motion from video sequences. The optical flow constraint is for- mulated in [11] and forms a basis for most of the later work on optical flow. Higher order structures in the optical flow may be inferred such as e.g. the optical acceleration [12], the structure of the motion parallax $[13,14]$ and polynomial approximation of same [15]. Various regularization of the measure flow has presented e.g. using a Bayes framework [16]. Earlier work on tangential regularization is presented in $[17,18]$. A thorough review of optical flow algorithms have been presented in $[19,20]$. The most effective algorithm to date is given in [21].

A major problem when inferring the apparent motion from video sequences is the aperture problem: only motion normal to isophotes may be measured. The work presented in the present article is for inferring the underlying flow from normal flows such as optical flow.

Almost all previous work considers normal flow alone. We take an outset from the normal flows and estimate a tangential counterpart. The purpose is to co-deform textures in morphing systems, and improve the estimate of the underlying physical flow in optical flow estimation. According to Weickert and Schnörr's taxonomy [22], our method is a homogeneous, isotropic, and image driven method with side information.

\section{Guessing Tangent Component by Energy Minimization}

We consider time varying vector fields on the form:

$V(x, t)=T(x, t)+N(x, t)$,

where $t \in \mathbb{R}_{+}, x \in \mathbb{R}^{D}, V, T, N: \mathbb{R}^{D} \times \mathbb{R}_{+} \rightarrow \mathbb{R}^{D}$, and where $T$ and $N$ are orthogonal vector fields,

$\langle T(x, t), N(x, t)\rangle=0$. 

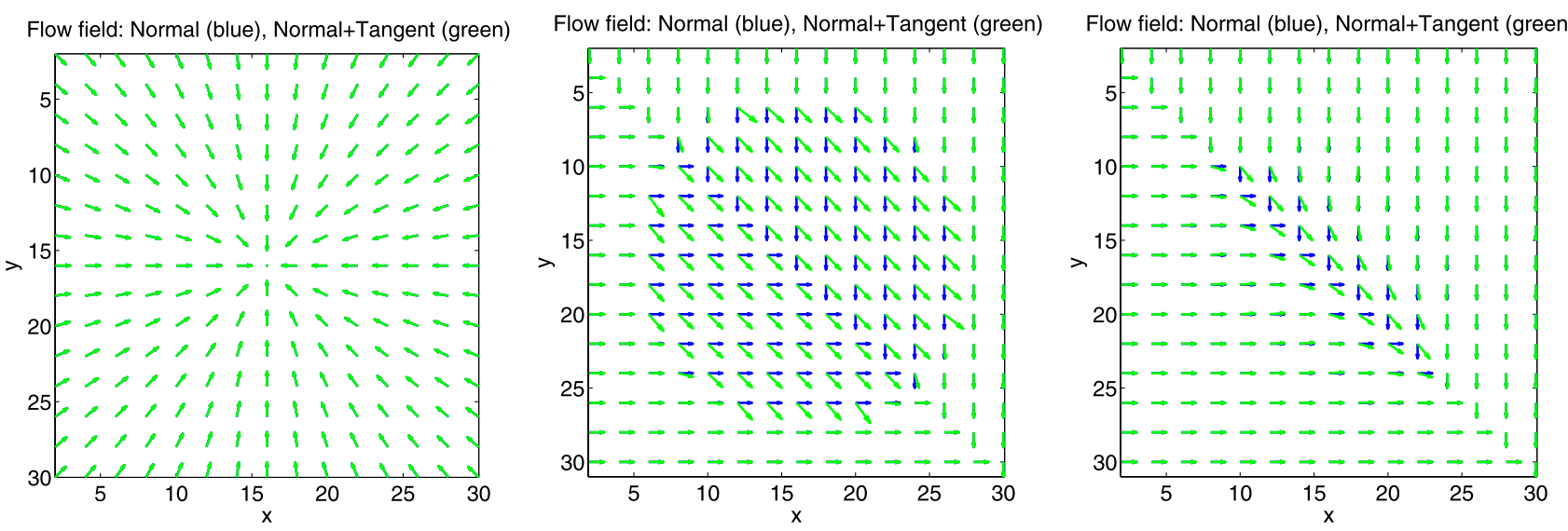

Fig. 3 (Color online) The result of our algorithm on two normal flows. The normal flow is shown in blue and the resulting flow by our method in green. Left is already at minimum, and Middle and Right shows the same Normal flow for a gradient type and a Laplacian type energy functional

Given $N(x, t)$ we wish to solve for $T(x, t)$ as the minimum of

$\mathcal{E}=\int_{\Lambda} \int_{\Omega} \sum_{i, j}\left\langle\mathcal{L}_{i} V(x, t), W_{i j}(x) \mathcal{L}_{j} V(x, t)\right\rangle \mathrm{d} x \mathrm{~d} t$,

for some suitable domain $\Lambda \times \Omega$, finite number of spatial operators on the class of smooth functions, $\mathcal{L}_{i}: \mathcal{C}\left(\mathbb{R}^{D} ; \mathbb{R}^{D}\right) \rightarrow$ $\mathcal{C}\left(\mathbb{R}^{D} ; \mathbb{R}^{D}\right)$, and weight matrices $W_{i j}: \mathbb{R}^{D} \rightarrow \mathbb{R}^{D \times D}$. The inner product, or squared two-norm, allows for a probabilistic interpretation [5] as a Gaussian distribution, $\mathcal{P}=$ $\frac{1}{Z} \exp (-\mathcal{E})$, where $Z$ is the normalization constant. Since $W_{i j}$ is predefined, this is a homogeneous, isotropic, and image driven method according [22] but with $N$ as side information.

To find the minimum of (3) as a function of the tangent vector field $T(x, t)$, we will use the Euler-Lagrange formulation, but for mathematical convenience we reduce the problem. Firstly, since the $\mathcal{L}_{i}$ 's are independent on time, then $\varepsilon$ is minimal, when

$\mathcal{E}(t)=\int_{\Omega} \sum_{i, j}\left\langle\mathcal{L}_{i} V(x, t), W_{i j}(x) \mathcal{L}_{j} V(x, t)\right\rangle \mathrm{d} x$,

is minimized separately for each $t \in \Lambda$. Secondly, for simplicity we choose spatially varying but isotropic norms,

$W_{i j}(x)=w_{i j}(x) \mathbf{1}$,

where $w_{i j}: \mathbb{R}^{D} \rightarrow[0, \infty)$ with $\inf _{x \in \Omega_{0}} w_{i j}(x)>0$ for every closed subset $\Omega_{0}$ of the interior of $\Omega$, and $\mathbf{1}$ is the identity matrix. The adjoint operator ${ }^{\dagger}$ is defined as, $\langle v, \mathcal{L} u\rangle=$ $\left\langle\mathcal{L}^{\dagger} v, u\right\rangle$. Hence, moving the weight function inside the inner product, writing $\mathcal{L}_{i}^{\dagger}$ as the adjoint of $\mathcal{L}_{i}$, and assuming that $w_{i j}(x)$ is zero on the boundary of the domain, we find

$$
\begin{aligned}
\mathcal{E} & =\int_{\Omega} \sum_{i, j}\left\langle\mathcal{L}_{j}^{\dagger}\left(w_{i j}(x) \mathcal{L}_{i} V(x, t)\right), V(x, t)\right\rangle \mathrm{d} x \\
& =\int_{\Omega}\langle\mathcal{K} V(x), V(x)\rangle \mathrm{d} x,
\end{aligned}
$$

where the operator $\mathcal{K}$ is given by,

$\mathcal{K} V(x)=\sum_{i, j} \mathcal{L}_{j}^{\dagger}\left(w_{i j}(x) \mathcal{L}_{i} V(x)\right)$.

We will in the following give 4 examples of the calculation of $\mathcal{K}$ from $\mathcal{L}$.

Constant $\mathcal{L}:$ In the simplest case,

$$
\mathcal{L}_{0} V(x)=c V(x),
$$

where $c>0$, the energy $\mathcal{E}$ is given as,

$$
\begin{aligned}
\int_{\Omega}\left\langle w_{00} \mathcal{L}_{0} V, \mathcal{L}_{0} V\right\rangle \mathrm{d} x & =\int_{\Omega}\left\langle w_{00} c V, c V\right\rangle \mathrm{d} x \\
& =\int_{\Omega}\left\langle w_{00} c^{2} V, V\right\rangle \mathrm{d} x,
\end{aligned}
$$

corresponding to a smoothness term penalizing $\|V\|^{2}$. This is minimized by $N$, but for expository reasons we conclude that $\mathcal{L}_{0}^{\dagger}=c$, and

$$
\mathcal{K} V(x)=w_{00}(x) c^{2} V(x)
$$

Gradient type $\mathcal{L}$ : For the more general case of constant and first order derivatives,

$$
\mathcal{L}_{0} V(x)=c V(x), \quad \mathcal{L}_{i} V(x) \stackrel{i=1, \ldots, D}{=} \partial_{i} V(x),
$$

where $c>0$. Choosing $w_{i j}(x)=1_{i=j} w(x)$, we find a weak string/membrane [23] smoothing term penalizing 
$\|\nabla u\|^{2}$, where $c$ determines the degree of smoothing. The operator $\mathcal{K}$ is evaluated to be,

$$
\begin{aligned}
\mathcal{K} V(x)= & c^{2} w(x) V(x)-\sum_{i=1}^{D} \partial_{i}\left(w(x) \partial_{i} V(x)\right) \\
= & c^{2} w(x) V(x)-\sum_{i=1}^{D} \partial_{i} w(x) \partial_{i} V(x) \\
& -w(x) \sum_{i=1}^{D} \partial_{i}^{2} V(x) \\
= & c^{2} w(x) V(x)-\left\langle\nabla_{x} w(x), \nabla_{x} V(x)\right\rangle \\
& +w(x) \Delta V(x) .
\end{aligned}
$$

Laplacian type $\mathcal{L}$ : For a system of constant and second order operators,

$\mathscr{L}_{0} V(x)=c V(x), \quad \mathscr{L}_{\Delta} V(x)=\Delta V(x)$,

where $c>0$. Choosing $w_{i j}(x)=1_{i=j} w(x)$, we find a weak rod/plate [23] smoothing term penalizing $\|\Delta V\|^{2}$, where the amount of smoothing is controlled by $c$. To evaluate the operator $\mathcal{K}$, we use that the Laplace operator $\Delta$ is self-adjoint $\Delta=\Delta^{\dagger}$, and we find,

$$
\mathcal{K} V(x)=c^{2} w(x) V(x)+\Delta(w(x) \Delta V(x)) .
$$

Statistical invariance type $\mathcal{L}$ : The general form of the weight matrices $W_{i j}: \mathbb{R}^{D} \rightarrow \mathbb{R}^{D \times D}$ come into play, when we want to use energies related to statistical invariant velocity fields in the sense of [24]. Assume that the velocity field $V: \mathbb{R}^{D} \rightarrow \mathbb{R}^{D}$ is a Gaussian random function with stationary increments, i.e. the distribution of $V(x)-V(y)$ only depends on $x, y \in \mathbb{R}^{D}$ via $x-y$. Then the results of [24] imply that

$$
\begin{aligned}
& \operatorname{cov}\left(\partial_{1}^{\alpha_{1}} \cdots \partial_{D}^{\alpha_{D}} V(x), \partial_{1}^{\beta_{1}} \cdots \partial_{D}^{\beta_{D}} V(x)\right) \\
& \quad=(-1)^{\sum_{i=1}^{D}\left(\alpha_{i}-\beta_{i}\right)} \operatorname{var}\left(\partial_{1}^{\frac{\alpha_{1}+\beta_{1}}{2}} \cdots \partial_{D}^{\frac{\alpha_{D}+\beta_{D}}{2}} V(x)\right),
\end{aligned}
$$

if the sums $\alpha_{i}+\beta_{i}$ of the differentiation orders are even for every $i=1, \ldots, D$, and that the covariance on the left hand side of (15) vanishes otherwise. If $\mathcal{L}_{\alpha}$ for $\alpha=\left(\alpha_{1}, \ldots, \alpha_{D}\right)$ denotes the differential operator $\partial_{1}^{\alpha_{1}} \cdots \partial_{D}^{\alpha_{D}}$, then the covariance structure described in (15) leads to the matrices

$$
\begin{aligned}
& \left\{W_{\alpha, \beta}(x)\right\}_{\alpha, \beta} \\
& \quad=\left\{(-1)^{\sum_{i=1}^{D}\left(\alpha_{i}-\beta_{i}\right)} 1_{\alpha_{1}+\beta_{1}, \ldots, \alpha_{D}+\beta_{D}}\right. \text { are even }
\end{aligned}
$$

$$
\left.\times \Gamma_{\left(\alpha_{1}+\beta_{1}, \ldots, \alpha_{D}+\beta_{D}\right)}\right\}_{\alpha, \beta}^{-1} w(x),
$$

where the $\Gamma$ 's are a set of parameters and $w(x)$ is the weight function taking care of the boundary conditions. We refer to [24] for a detailed analysis of statistical invariance properties of vector fields.

\section{Invariance Properties}

In the following we will discuss algebraic invariance of vector fields. Statistical invariance properties are discussed in an accompanying article [24].

Consider a Gaussian type window functions,

$w^{n}(x)=\exp \left(-\frac{1}{2}\left(\frac{x^{2}+y^{2}}{\sigma^{2}}\right)^{n}\right)$,

for some given $n$, and as an example consider the gradient type operator discussed above, applied to a linear vector field,

$V(x, y)=\left[\begin{array}{l}a_{0}+a_{1} x+a_{2} y \\ b_{0}+b_{1} x+b_{2} y\end{array}\right]$.

Applying (12), we find that

$$
\begin{aligned}
\mathcal{K} V(x, y)= & c^{2} w(x, y) V(x, y) \\
& -\left[\begin{array}{ll}
a_{1} & a_{2} \\
b_{1} & b_{2}
\end{array}\right]\left[\begin{array}{l}
w_{x}(x, y) \\
w_{y}(x, y)
\end{array}\right] .
\end{aligned}
$$

In the limit of $c \rightarrow 0$ and $n \rightarrow \infty$, the operator $\mathcal{K} V(x, y)$ will be zero except at the border of the window, where $x^{2}+y^{2}=\sigma^{2}$. The conclusion is that the Euler-Lagrange derivative for Gradient type operators is zero for fields of degree less than two except at the border at $x^{2}+y^{2}=\sigma^{2}$, where $w(x, y) V(x, y)$ acts as border conditions, and thus $V$ is an essential fixpoint for the gradient type energy functionals.

This result can be generalized: Any operator $\mathcal{L}=\partial^{m}$, $m>0$, will result in an operator $\mathcal{K}=(-1)^{m} \partial^{m}\left(w(x) \partial^{m} \times\right.$ $V(x))$. Thus we conclude, that for an analytical vector field $V$ and in the limit of $c \rightarrow 0$ and $n \rightarrow \infty$, the operator $\mathcal{K}$ will be invariant to terms in $V$ of order less than $m^{2}$ except at the border of the window, where $w(x, y) V(x, y)$ acts as a border condition.

\section{Solving Energy by Euler-Lagrange Equations}

Once a set of operators $\mathcal{L}_{i}$ has been chosen, and the corresponding $\mathcal{K}$ has been calculated, the energy formulation is ready to be minimized. Remembering (1) and (2) we 
have that $V(x, t)=T(x, t)+N(x, t)$, and that our solution is to be found in the subspace orthogonal to $N(x, t)$, $\langle T(x, t), N(x, t)\rangle=0$. Thus we combine the energy and the constraint into a Lagrange equation,

$$
\begin{aligned}
E= & \int_{\Omega}\langle\mathcal{K} V(x), V(x)\rangle \mathrm{d} x \\
& -\int_{\Omega} \lambda(x)\langle T(x), N(x)\rangle \mathrm{d} x,
\end{aligned}
$$

and use the variational method to simultaneously solve,

$$
\frac{\delta E}{\delta T(x)}=0, \quad \frac{\delta E}{\delta \lambda(x)}=0 .
$$

The first term of $E$ contains our variable field $T$ on both sides of the inner product. To find the solution, we may use the adjoint of $\mathcal{K}$ satisfying $\langle\mathcal{K} V(x), V(x)\rangle=$ $\left\langle V(x), \mathcal{K}^{\dagger} V(x)\right\rangle$, such that when we find the derivative wrt. the right $V$ we use the left form and vice-versa. To simplify matters further, $\mathcal{K}$ is self-adjoint, i.e. $\mathcal{K}=\mathcal{K}^{\dagger}$, which may be seen by expanding $\mathcal{K}$ and moving terms from one side of the inner product to the other. Using the above and superscript to denote placeholders, we find the Euler-Lagrange equations (21) to be,

$$
\begin{aligned}
0= & \frac{\delta E}{\delta T(x)} \\
= & \left.\frac{\delta \int_{\Omega}\left\langle\mathcal{K} V^{1}(x), V^{2}(x)\right\rangle \mathrm{d} x}{\delta V^{1}(x)} \frac{\delta V^{1}(x)}{\delta T(x)}\right|_{V^{1}=V^{2}=V} \\
& +\left.\frac{\delta \int_{\Omega}\left\langle\mathcal{K} V^{1}(x), V^{2}(x)\right\rangle \mathrm{d} x}{\delta V^{2}(x)} \frac{\delta V^{2}(x)}{\delta T(x)}\right|_{V^{1}=V^{2}=V} \\
& -\frac{\delta \int_{\Omega} \lambda(x)\langle T(x), N(x)\rangle \mathrm{d} x}{\delta T(x)} \\
= & \left.\frac{\delta \int_{\Omega}\left\langle V^{1}(x), \mathcal{K} V^{2}(x)\right\rangle \mathrm{d} x}{\delta V^{1}(x)}\right|_{V^{1}=V^{2}=V} \\
& +\mathcal{K} V(x)-\lambda(x) N(x) \\
= & 2 \mathcal{K} V(x)-\lambda(x) N(x) .
\end{aligned}
$$

The conclusion is that $\mathcal{K} V(x)$ is parallel to $N(x)$. Introducing an orthonormal decomposition of the tangent space as,

$T(x)=\sum_{b=1}^{D-1} T_{b}(x) \mu_{b}(x)$,

where $T_{b}(x) \in \mathbb{R}^{D}$ with $\left\|T_{b}(x)\right\|=1, \mu_{b}(x) \in \mathbb{R}, b=$ $1, \ldots, D-1$, the fact that $\mathcal{K} V(x)$ is parallel to $N(x)$ may be used directly in the orthogonality of the tangent space to the normal (2), which gives us the following equations,

$0=\left\langle T_{b}(x), N(x)\right\rangle=\left\langle T_{b}(x), \mathcal{K} V(x)\right\rangle$,

$$
b=1, \ldots, D-1 .
$$

For the gradient type examples this system of equations is an elliptical second order partial differential equations, where $N(x) \neq 0$, and such equations are simple to implement using standard numerical methods as described in e.g. [25].

\section{Numerical Solution by Parabolic Equivalent}

To avoid inverting large matrix systems, a standard alternative is to seek the solution to an elliptical type partial differential equation as the limit of a parabolic type differential equation by introducing an artificial time, and evaluating the result at infinity (or a very large iteration number). We adopt this general principle by introducing $\tau$ as time, and we write the non-optimal field of $\mu_{b, \tau}(x)$ and the non-optimal vector field $V_{\tau}$ as,

$V_{\tau}(x)=\sum_{b=1}^{D-1} T_{b}(x) \mu_{b, \tau}(x)+N(x)$.

The iteration-time derivative of $V_{\tau}$ is given as,

$\frac{\partial V_{\tau}(x)}{\partial \tau}=\sum_{b=1}^{D-1} T_{b}(x) \frac{\partial \mu_{b, \tau}(x)}{\partial \tau}$,

and we propose to seek the solution by letting,

$\frac{\partial \mu_{b, \tau}(x)}{\partial \tau}=-\left\langle T_{b}(x), \mathcal{K} V_{\tau}(x)\right\rangle$.

The operator $\mathcal{K}$ is said to be coercive on the subdomain $\Omega_{0} \subseteq \Omega$, if there exists $c>0$, such that $\langle U(x), \mathcal{K} U(x)\rangle \geq$ $c^{2}\|U(x)\|^{2}$ for every vector field $U(x)$ and every $x \in \Omega_{0}$. If $\mathcal{L}_{0} V(x)=c V(x)$ is included in the list of spatial operators, and $w_{00}(x)$ only vanishes on the boundary of $\Omega$, then $\mathcal{K}$ is coercive on every closed subset $\Omega_{0}$ of the interior of $\Omega$. For such a $\mathcal{K}$ we will now prove that (27) leads to a set of parameters $\mu_{b} T_{b}$, which are perpendicular to $\mathcal{K} V(x)$, hence a solution to (21).

Proof We seek $\left\langle T_{b}(x), \mathcal{K} V(x)\right\rangle=0$ using $\frac{\partial \mu_{b, \tau}(x)}{\partial \tau}=$ $-\left\langle T_{b}(x), \mathcal{K} V_{\tau}(x)\right\rangle$ : Let $\Omega_{0}$ be a closed subset of the interior of $\Omega$, and choose $c>0$ such that $\langle U(x), \mathcal{K} U(x)\rangle \geq$ $c^{2}\|U(x)\|^{2}$ for every $x \in \Omega_{0}$. We have

$$
\begin{aligned}
\frac{1}{2} & \frac{\partial}{\partial \tau} \sum_{b=1}^{D-1} \int_{\Omega_{0}}\left\|\left\langle T_{b}(x), \mathcal{K} V_{\tau}(x)\right\rangle\right\|^{2} \mathrm{~d} x \\
& =\sum_{b=1}^{D-1} \int_{\Omega_{0}}\left\langle T_{b}, \mathcal{K} V_{\tau}\right\rangle^{\top}\left\langle T_{b}, \frac{\partial \mathcal{K} V_{\tau}}{\partial \tau}\right\rangle \mathrm{d} x
\end{aligned}
$$




$$
\begin{aligned}
&= \int_{\Omega_{0}} \sum_{b=1}^{D-1}\left\langle T_{b}, \mathcal{K} V_{\tau}\right\rangle^{\top} \\
& \times\left\langle T_{b}, \mathcal{K}\left(\sum_{d=1}^{D-1} T_{d} \frac{\partial \mu_{d, \tau}}{\partial \tau}\right)\right\rangle \mathrm{d} x \\
&= \int_{\Omega_{0}}\left\langle\sum_{b=1}^{D-1} T_{b}\left\langle T_{b}, \mathcal{K} V_{\tau}\right\rangle, T_{d},\right. \\
& \leq-c^{2} \sum_{b=1}^{D-1} \int_{\Omega_{0}}\left\|\left\langle T_{b}(x), \mathcal{K} V_{\tau}(x)\right\rangle\right\|^{2} \mathrm{~d} x,
\end{aligned}
$$

and Grönwall's inequality [26] gives

$$
\begin{aligned}
& \sum_{b=1}^{D-1} \int_{\Omega_{0}}\left\|\left\langle T_{b}(x), \mathcal{K} V_{\tau}(x)\right\rangle\right\|^{2} \mathrm{~d} x \\
& \quad \leq \text { const. } \cdot \exp \left(-2 \tau c^{2}\right) .
\end{aligned}
$$

We conclude that $\left\langle T_{b}(x), \mathcal{K} V_{\tau}(x)\right\rangle \rightarrow 0$ as $\tau \rightarrow \infty$ for every $x$ in the interior of $\Omega$.

\section{Results on Synthetic Motion Fields}

In the remainder of this article we will give examples of minimal vector fields for various prototypical normal fields and parameter settings. The order of the operator implies fixpoints in the Euler-Lagrange equations, such that first order vector fields are fixpoints for first order operators up to boundary conditions and zero order regularization and likewise for third order vector fields and second order operators. This will be demonstrated in the following for two dimensional fields, where $(x, y)$ will denote the coordinates. As discussed in (24), the Euler-Lagrange equation is optimal when $N(x, y)$ is parallel to $\mathcal{K} V(x, y)$. Hence for fixpoints we must have that

$$
\mathcal{K} V(x, y) \times V(x, y)=0 .
$$

In the following we will limit ourselves to show general vector fields which fulfills this equation by $\mathcal{K} V(x, y)=0$. In our experiments we have used Gaussian type window functions,

$w^{n}(x)=\exp \left(-\frac{1}{2}\left(\frac{x^{2}+y^{2}}{\sigma^{2}}\right)^{n}\right)$,

for some given $n$. In this and the remaining examples we have used $\sigma=M / 4$ for vector fields on an $M \times M$ domain.
6.1 Gradient Type

The regularized gradient type operator is defined using three independent operators,

$$
\begin{aligned}
& \mathcal{L}_{0} V(x, y)=c V(x, y), \\
& \mathcal{L}_{1} V(x, y)=\partial_{x} V(x, y), \\
& \mathcal{L}_{2} V(x, y)=\partial_{y} V(x, y),
\end{aligned}
$$

and we have shown in (12) that

$$
\begin{aligned}
\mathcal{K} V(x, y)= & c^{2} w(x, y) V(x, y)-\left\langle\nabla_{x} w(x, y), \nabla_{x} V(x, y)\right\rangle \\
& +w(x, y) \Delta V(x, y) .
\end{aligned}
$$

The first term is due to the zeroth order regularizor, the second term as consequence of our border condition, and third term as a result of first order regularizors.

To solve the Euler-Lagrange equation by (27) we have written a program, which implements von Neumann boundary conditions at the image border, and is designed to terminate as soon as the first minimal energy value is observed. Near invariant flow fields are generated as the gradient of second degree polynomials for a source and saddle type divergent field, and by hand as a curl field. These and their solutions are shown in Fig. 4. The normal vector field and the minimal counterpart is shown in blue and green respectively, we have used a window of exponent $n=4$, and regularization constant $c=0.01$. The fields are observed to be essentially invariant to the gradient type functional.

A non-trivial example is shown in Fig. 5. This vector field has a singular line as produced by the superposition of the optical flow observed, when a box structure is moved diagonally down and to the right. The right images display the values of $\mu_{b}$ in each pixel, and the energy plot. We conclude that the minimal energy is reached, and that the point of minimum energy coincides with the interpretation of the diagonal motion in spite that only the normal component is given.

Changing window exponent influences the focus of the method. The result of running our method on the singular line example with 3 different exponents is shown in Fig. 6. The numerical implementation does not seem to be disturbed by the fact that low order exponents results in defacto non-zero values of the window at the border. We attribute this result to the von Neumann border conditions.

Various values of the regularization constant $c$ on the singular line problem is shown in Fig. 7. Large values of $c$ implies that the resulting vector field is close to the original normal field, while low values allow for the gradient term to be dominating. 

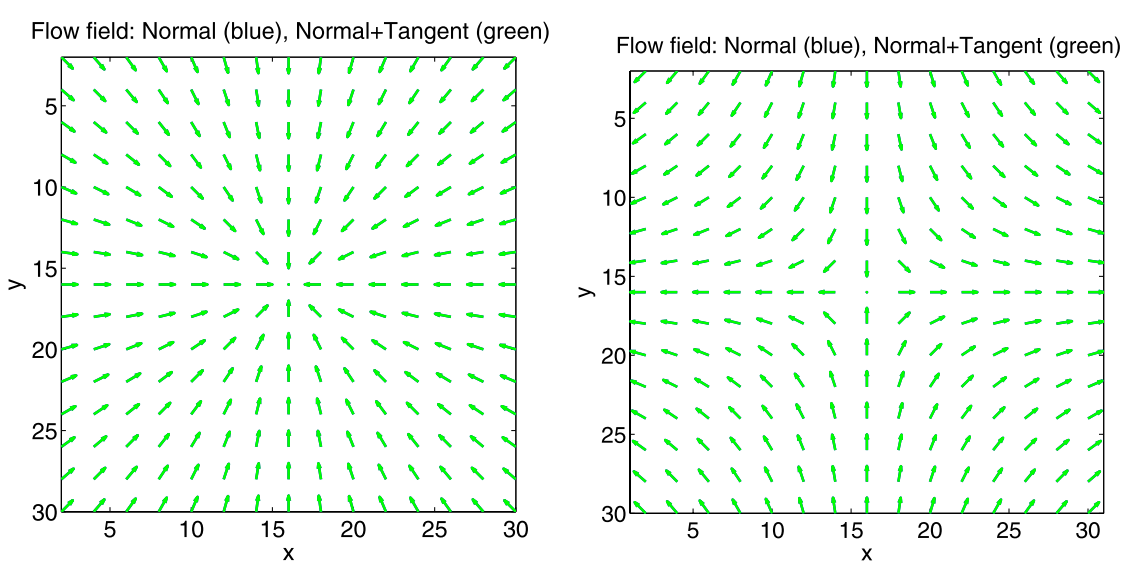

Flow field: Normal (blue), Normal+Tangent (green)

Fig. 4 (Color online) Different normal flows and their minimum for Gradient type functionals. Left and middle are gradient fields of a second degree polynomial for a minimum and a saddle respectively, and right shows a curl field. We have used $c=0.01$ and $n=4$

Fig. 5 (Color online)

Minimizing Ridge normal flow with gradient type operators. Left shows the original and resulting flow, and right shows the resulting tangential parameter, and the energy evolution during minimization
Flow field: Normal (blue), Normal+Tangent (green)

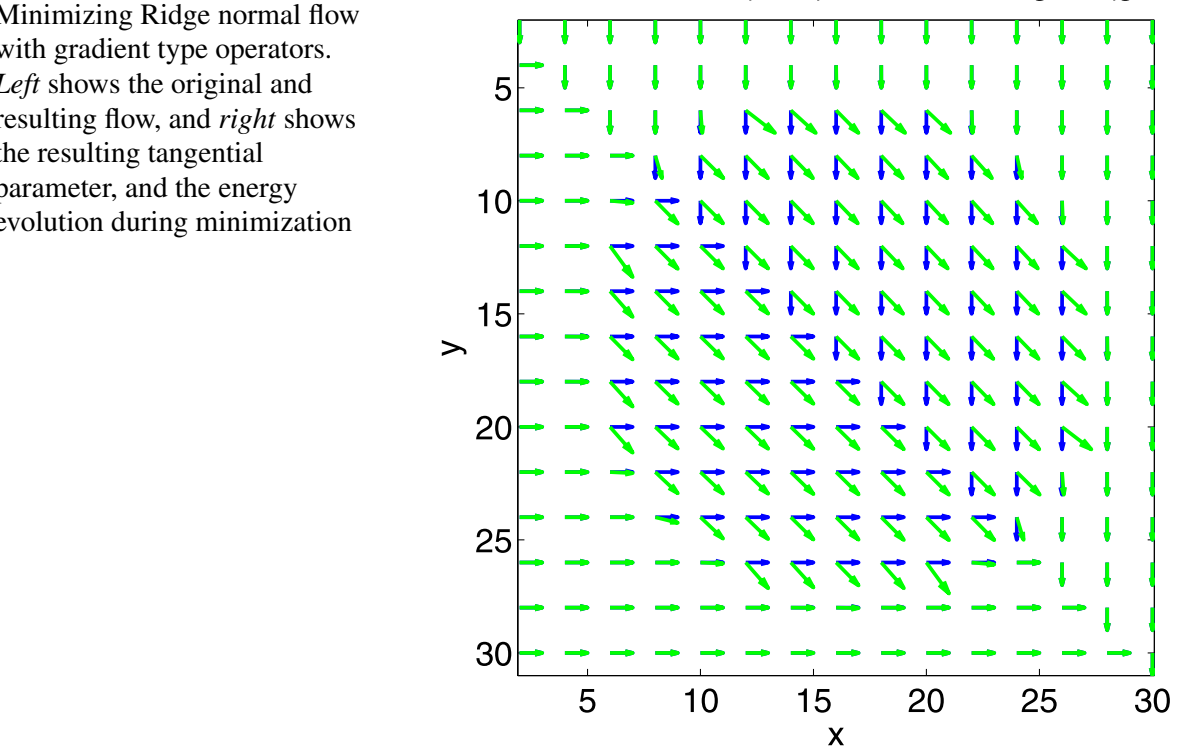

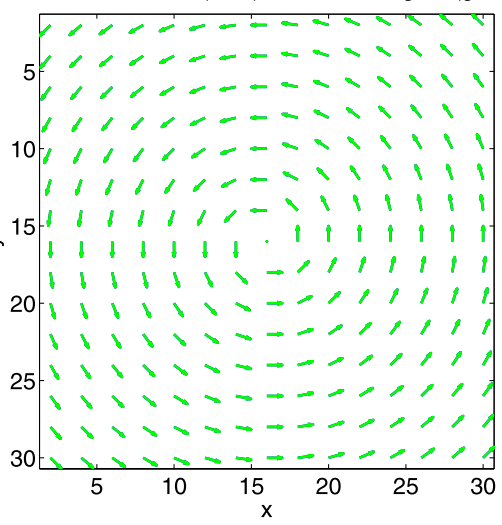
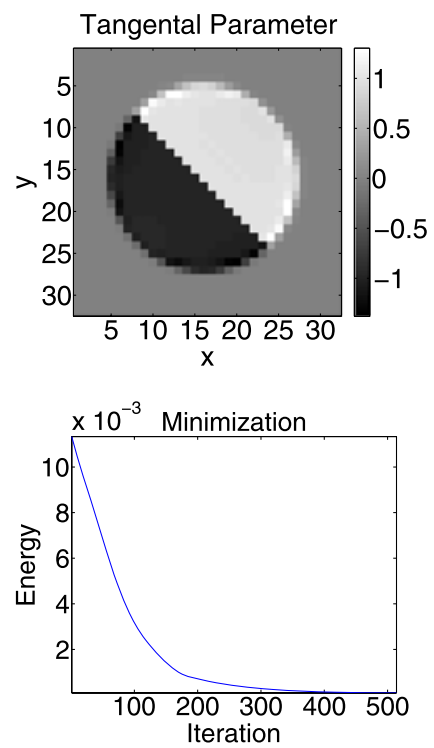

Flow field: Normal (blue), Normal+Tangent (green)

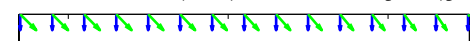

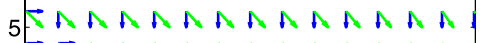
$\rightarrow R N A N A N A N A N A N$ $\rightarrow \nabla R A N A N A N A \Lambda A N$ $10-\nabla \nabla \wedge \wedge \wedge \wedge \wedge \wedge \wedge \wedge \Lambda \wedge$

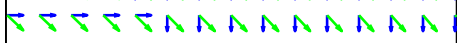
$15 \vec{\nabla} \nabla \nabla \nabla N \wedge N \wedge N \wedge N \wedge$

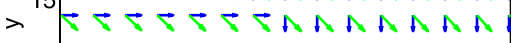

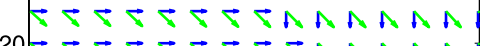

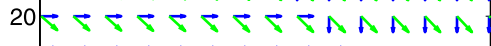

$\rightarrow \nabla \nabla \nabla \nabla R \nabla R$

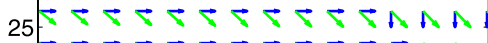

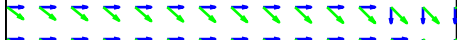

$\rightarrow r \rightarrow \vec{r} \rightarrow \vec{r} \rightarrow \vec{r}+\vec{r}$

\begin{tabular}{rlllll}
\hline 5 & 10 & 15 & 20 & 25 & 30
\end{tabular}
Flow field: Normal (blue), Normal+Tangent (green)

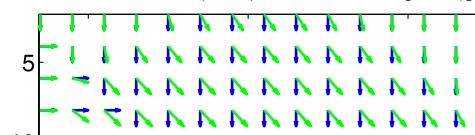
$10-\nabla \nabla N \wedge N \Lambda N N \Lambda N \Lambda N$

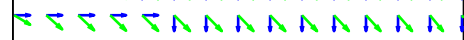

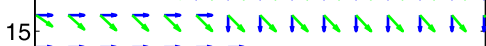

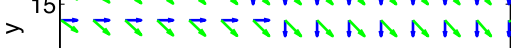
$\rightarrow \nabla \nabla \nabla \nabla \nabla N \wedge N \wedge N$

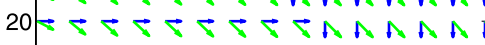
$\rightarrow \nabla \nabla \nabla \nabla R+\nabla N \Lambda$

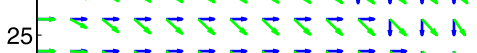

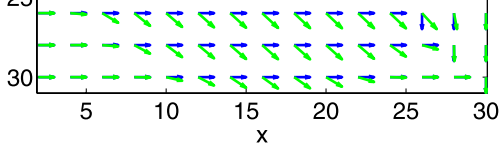

Flow field: Normal (blue), Normal+Tangent (green)

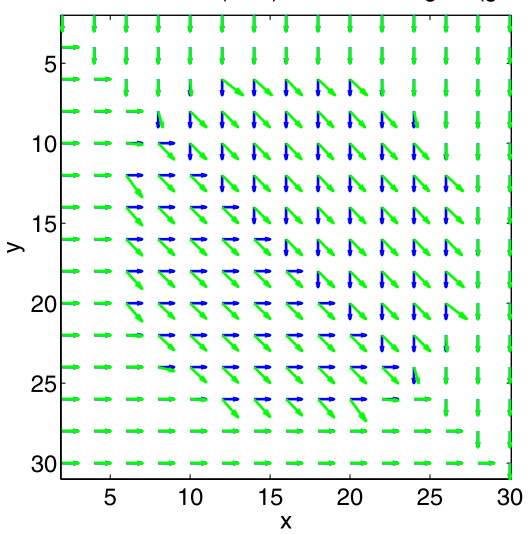

Fig. 6 (Color online) Result of varying exponent in Gaussian type window. From left to right: $n=1,2,4$ 

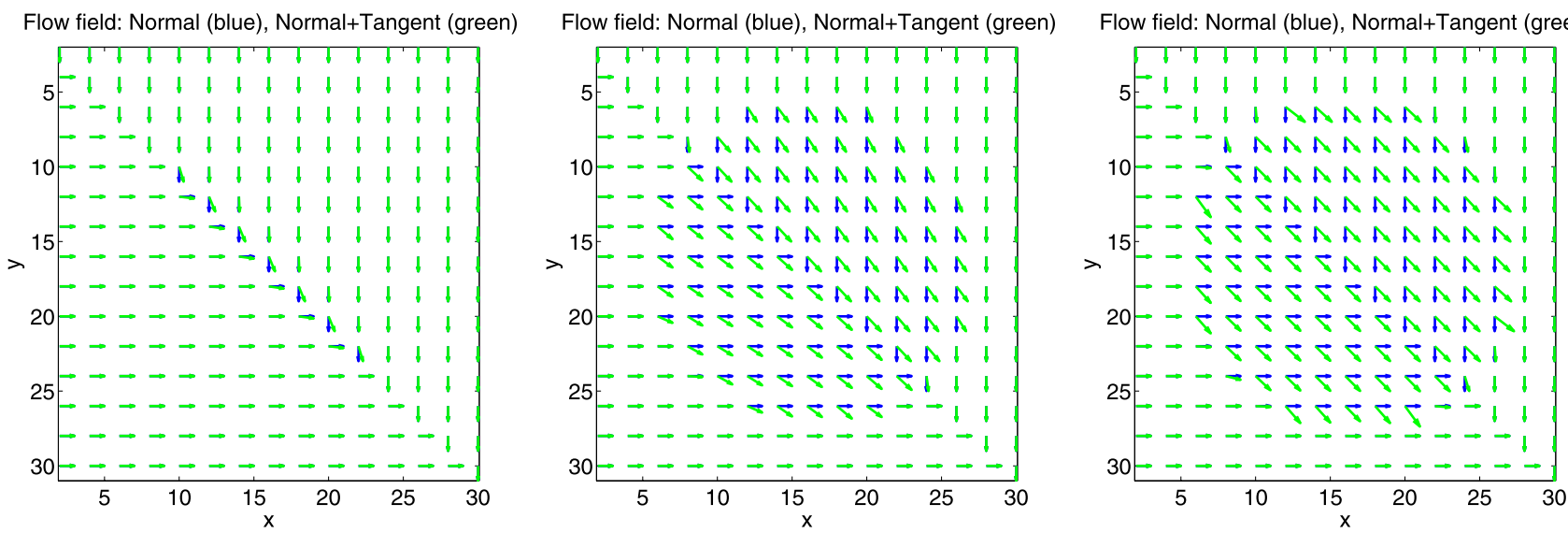

Fig. 7 (Color online) Result of varying regularization constant $c$. From left to right: $c=1,0.1,0.01$

Fig. 8 (Color online) Minimizing Ridge normal flow with Laplace type operators. Left shows the original and resulting flow, and right shows the resulting tangential parameter, and the energy evolution during minimization
Flow field: Normal (blue), Normal+Tangent (green)

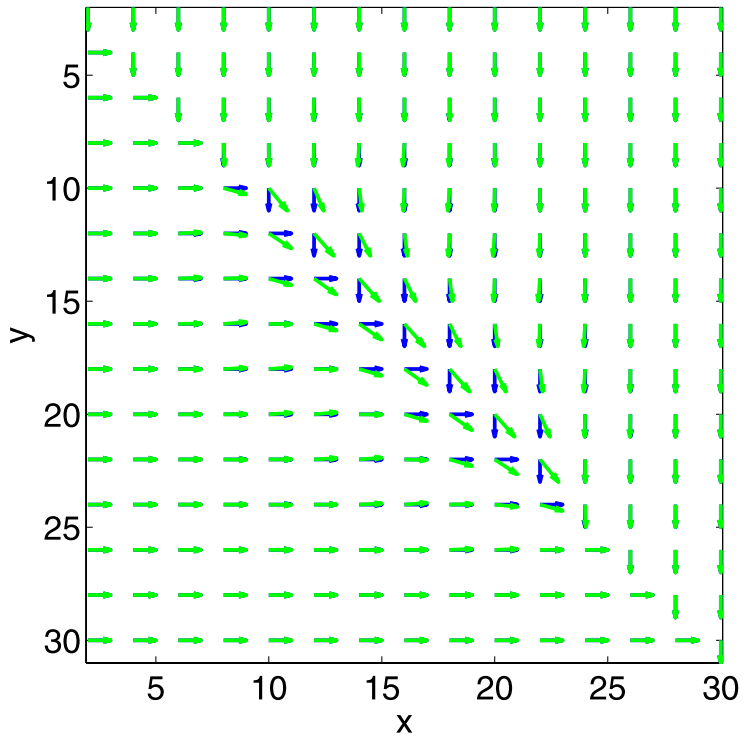

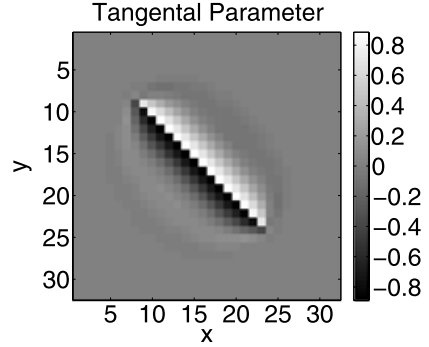

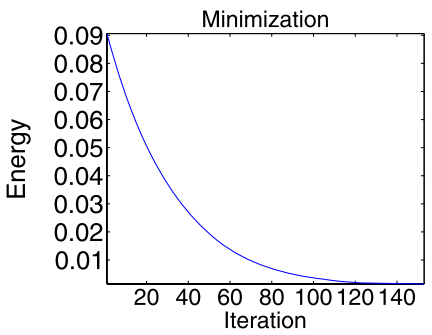

\subsection{Laplace Type}

We have defined a regularized Laplacian type by two independent operators,

$$
\begin{aligned}
& \mathcal{L}_{0} V(x, y)=c V(x, y), \\
& \mathcal{L}_{1} V(x, y)=\Delta V(x, y),
\end{aligned}
$$

where $c>0$, which by (14) was seen to be equivalent to

$$
\begin{aligned}
\mathcal{K} V(x, y)= & c^{2} w(x, y) V(x, y) \\
& +\Delta(w(x, y) \Delta V(x, y)) \\
= & c^{2} w(x, y) V(x, y)+\Delta w(x, y) \Delta V(x, y) \\
& +2\left(\nabla_{x} \Delta V(x, y)\right) \nabla_{x} w(x, y) \\
& +w(x, y) \Delta \Delta V(x, y) .
\end{aligned}
$$

Using the same argument as from the Gradient type operator we conclude, that when $c \rightarrow 0$ and $n \rightarrow \infty$, then $\mathcal{K} V(x, y) \rightarrow w(x, y) \Delta \Delta V(x, y)$ almost everywhere, and this is zero for vector fields of degree smaller than four. The effective border condition is again found at $x^{2}+y^{2}=\sigma^{2}$.

Low order normal vector fields are invariant under the Laplace type functionals as shown algebraically above, and solutions by our program on gradient fields of a second degree polynomial for a minimum, saddle respectively, and curl field for $c=0.01$ and $n=4$ are identical to Fig. 4. The Laplace type operators allows for more smoothly varying flow fields as shown on the singular line field in Fig. 8. In these experiments we have used $n=6$ and $c=0.01$. The influence of the window exponent on the singular line example is shown in Fig. 9. Compared to the Gradient type operators, these fields are more smoothly varying. The dependency on 

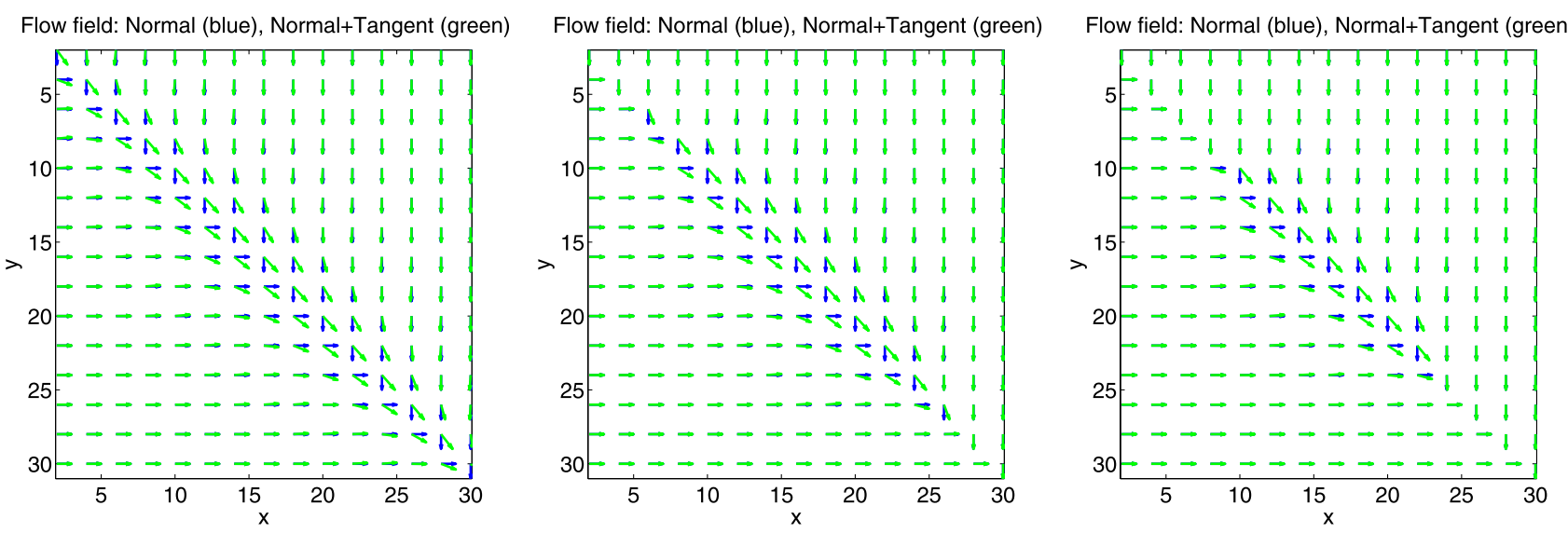

Fig. 9 (Color online) Result of varying exponent in Gaussian type window. From left to right: $n=1,2,4$
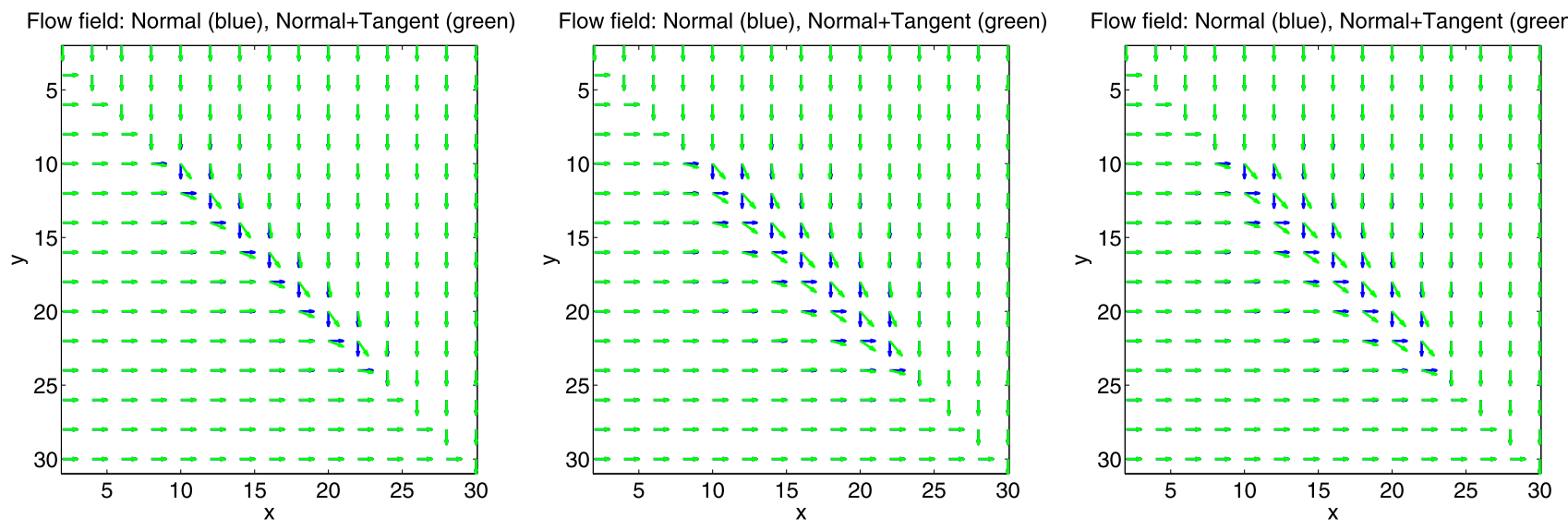

Fig. 10 (Color online) Result of varying regularization constant $c$. From left to right: $c=1,0.1,0.01$

the regularization constant $c$ on the singular line problem is shown in Fig. 10.

\section{Conclusion}

Several problems in Computer Vision and Graphics involve normal flows. The aperture problem in optical flow dictates that only the motion parallel to the isophote normal is observable in video sequences. However, the normal flow is a projection of the physical flow onto the isophote normals and may be far from the projection of the physical flow onto the image. The later being the important information in tracking applications etc. Likewise, shape-morphing is typically only concerned with shape deformations, and thus only expressed in the shape normals. However, since many shapes in computer graphics have textures, the warped texture will be unnatural and difficult to control in this manner.
In this paper we have extended the formalism for regularizing normal flows by an energy minimization technique on the inner product of spatial operators on the flow field. We have given a thorough mathematical description to great generality, we have presented a very simple, iterative, and parabolic-type algorithm, and we have proven convergence for this algorithm in the continuous setting.

The partial differential equations developed are not defined in points, where the normal flow vanishes. One solution could be to use the window function to mask out regions of vanishing normal flows. However, in the examples we have studied, we have not needed such interventions, which we attribute to the usual stability of parabolic schemes. Future work include, investigating examples in higher dimensional domains, producing coarse to fine continuation schemes to improve convergence rates, benchmark the method against existing optical flow algorithms, and further studies into the space of useful operators for computer vision and graphics problems. 


\section{References}

1. Nielsen, M., Andresen, P.: Feature displacement interpolation. In: IEEE 1998 International Conference on Image Processing (ICIP'98), pp. 208-212 (1998)

2. Joshi, S.C., Miller, M.I.: Landmark matching via large deformation diffeomorphisms. IEEE Trans. Image Process. 9, 1357-1370 (2000)

3. Christensen, G.E., Yin, P., Vannier, M.W., Chao, K.S.C., Dempsey, J.F., Williamson, J.F.: Large-deformation image registration using fluid landmarks. In: SSIAI Archive Proceedings of the 4th IEEE Southwest Symposium on Image Analysis and Interpretation, pp. 269-273 (2000)

4. Nielsen, M., Markussen, B.: From Bayes to PDEs in image warping. In: Faugeras, O., Paragios, N., Chen, Y. (eds.) Mathematical Models in Computer Vision: The Handbook, pp. 259-272. Springer, Berlin (2005), Chap. 16

5. Markussen, B.: Large deformation diffeomorphisms with application to optic flow. Comput. Vis. Image Underst. (2006, to appear)

6. Sethian, J.A.: Level Set Methods and Fast Marching Methods, 2nd edn. Cambridge University Press, Cambridge (1999)

7. Osher, S., Fedkiw, R.: Level Set Methods and Dynamic Implicit Surfaces. Springer, Berlin (2003)

8. Whitaker, R.T., Breen, D.E.: Level-set models for the deformation of solid objects. In: Saupe, D., Bloomenthal, J. (eds.) Implicit Surfaces 98 Proceedings, Eurographics/ACm Workshop, pp. 19-35 (1998)

9. Breen, D.E., Mauch, S., Whitaker, R.T., Mao, J.: 3d metamorphosis between different types of geometric models. Comput. Graph. Forum 20(3) (2001)

10. Breen, D.E., Whitaker, R.T.: A level-set approach for the metamorphosis of solid models. IEEE Trans. Vis. Comput. Graph. 7(2), 173-192 (2001)

11. Horn, B., Schunck, B.: Determining optical flow. Artif. Intell. 23, 185-203 (1981)

12. Arnspang, J.: Optic acceleration. In: International Conference on Computational Vision, pp. 364-373 (1989)

13. Koenderink, J.J., van Doorn, A.J.: Local structure of movement parallax of the plane. J. Opt. Soc. Am. 66(7), 717-723 (1976)

14. Sporring, J., Nielsen, M.: Direct estimation of first order optic flow. In: Borgefors, G. (ed.) Theory And Applications of Image Analysis II-Selected Papers from the 9th Scandinavian Conference on Image Analysis, pp. 225-238. World Scientific, Singapore (1995)

15. Pedersen, K.S., Nielsen, M.: Computing optic flow by scalespace integration of normal flow. In: Kerckhove, M. (ed.) ScaleSpace and Morphology in Computer Vision: Proceedings of Scale-Space'01. Lecture Notes in Computer Science, vol. 2106, pp. 14-25. Springer, Berlin (2001)

16. Weiss, Y., Adelson, E.H.: Slow and smooth: a Bayesian theory for the combination of local motion signals in human vision. Technical Report A.I. Memo No. 1624 and C.B.C.L. Paper No. 158, Massachusetts Institute of Technology, Artificial Intelligence Laboratory and Center for Biological and Computational Learning, Department of Brain and Cognitive Sciences (1998)

17. Nagel, H.-H., Enkelmann, W.: An investigation of smoothness constraints for the estimation of displacement vector fields from image sequences. IEEE Trans. Pattern Anal. Mach. Intell. 8, 565593 (1986)

18. Nagel, H.-H.: Extending the 'oriented smoothness constraint' into the temporal domain and the estimation of derivatives of optical flow. In: European Conference on Computer Vision-ECCV '90. Lecture Notes in Computer Science, vol. 427, pp. 139-148. Springer, Berlin (1990)

19. Barron, J.L., Fleet, D.J., Beauchemin, S.S.: Performance of optical flow techniques. Int. J. Comput. Vis. 12(1), 43-77 (1994)
20. Bruhn, A., Weickert, J., Schnörr, C.: Lucas/Kanade meets Horn/Schunck: Combining local and global optic flow methods. Int. J. Comput. Vis. 61(3), 211-231 (2005)

21. Brox, T., Bruhn, A., Papenberg, N., Weickert, J.: High accuracy optical flow estimation based on a theory for warping. In: Pajdla, T., Matas, J. (eds.) Proceedings of the 8th European Conference on Computer Vision, Prague, Czech Republic, vol. 4, pp. 25-36. Springer, Berlin (2004)

22. Weickert, J., Schnörr, C.: A theoretical framework for convex regularizers in pde-based computation of image motion. Int. J. Comput. Vis. 45, 245-264 (2001)

23. Blake, A., Zisserman, A.: Visual Reconstruction. MIT Press, Cambridge (1987)

24. Markussen, B., Pedersen, K.S., Loog, M.: Second order structure of scale-space measurements. J. Math. Imag. Vis. (2008, this issue)

25. Morton, K.W., Mayers, D.F.: Numerical Solution of Partial Differential Equations, 2nd edn. Cambridge University Press, Cambridge (2005)

26. Grönwal, T.H.: Note on the derivative with respect to a parameter of the solutions of a system of differential equations. Ann. Math. 20, 292-296 (1919)

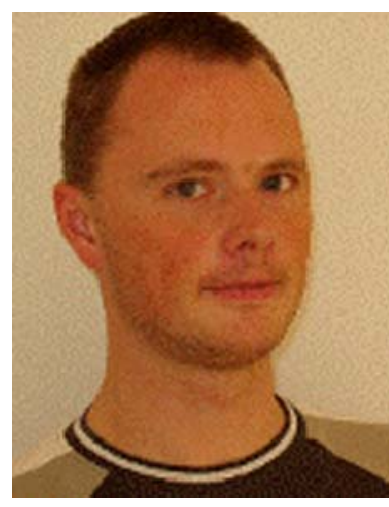

Bo Markussen received the Cand. Scient and Ph.d. degrees in mathematical statistics from University of Copenhagen, Denmark. From November 2001 to September 2003 he worked as post doc in Copenhagen and Berlin, and from October 2003 to December 2005 he was enrolled in the "Computing Natural Shape" project at the Department of Computer Science, University of Copenhagen. In January 2006 he joined the Deparment of Natural Sciences at the Royal Veterinary and Agricultural University (KVL), Denmark, as an Assistent Professor of statistics. KVL has now been merged into the University of Copenhagen as the Faculty of Life Sciences. The research interests of Bo Markussen includes applied statistics, stochastic calculus and applications to image analysis.

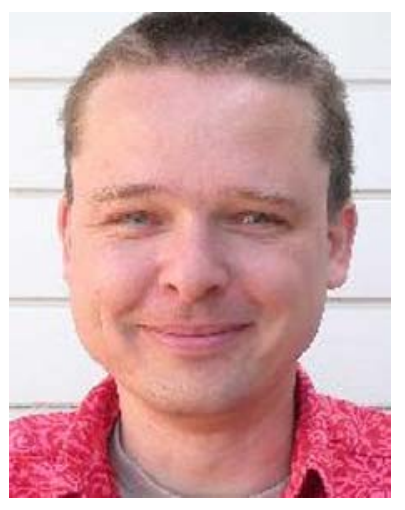

Jon Sporring received his Master and Ph.D. degree from the Department of Computer Science, University of Copenhagen, Denmark in 1995 and 1998, respectively. Part of his Ph.D. program was carried out at IBM Research Center, Almaden, California, USA. Following his Ph.D, he worked as a visiting researcher at the Computer Vision and Robotics Lab at Foundation for Research \& Technology Hellas, Greece, and as assistant research professor at 3D-Lab, School of Dentistry, University of Copenhagen. Since 2003 he has been employed as associate professor at the Department of Computer Science, University of Copenhagen, and since 2008 he has been Vice-Chair for Research at Department of Computer Science. His main topics of research are medical image processing, medical computer graphics, and information theory. 


Kenny Erleben After his studies Er-
leben was employed as full time re-
searcher in the Company 3DFacto
A/S for a period of 10 months. In
2001 Erleben started on his Ph.D.
studies. During 2004 Erleben stayed
3 months at the Department of Math-
ematics, University of Iowa. Here-
after he received his PhD degree in
the begining of 2005 and finally late
2005 Erleben was employed as an
Assistant Professor at the Depart-
ment of Computer Science, Univer-
sity of Copenhagen.

